\section{OPEN ACCESS}

Edited by:

Rui-Pin Chen,

Zhejiang Sci-Tech University, China

Reviewed by:

Bing $\mathrm{Gu}$,

Southeast University, China

Qin Wang,

Nanjing University of Posts and

Telecommunications, China

Ming Kang,

Tianjin Normal University, China

*Correspondence:

Zhi-Han Zhu

zhuzhihan@hrbust.edu.cn

Bao-Sen Shi

drshi@ustc.edu.cn

Specialty section:

This article was submitted to

Optics and Photonics,

a section of the journal

Frontiers in Physics

Received: 16 January 2021 Accepted: 01 March 2021

Published: 22 April 2021

Citation:

Wu H-J, Yu B-S, Zhu Z-H,

Rosales-Guzmán C, Zhou Z-Y,

Ding D-S, Gao W and Shi B-S (2021)

Heralded Generation of Vectorially Structured Photons With a High Purity.

Front. Phys. 9:654451.

doi: 10.3389/fphy.2021.654451

\title{
Heralded Generation of Vectorially Structured Photons With a High Purity
}

\author{
Hai-Jun Wu ${ }^{1}$, Bing-Shi Yu ${ }^{1}$, Zhi-Han Zhu ${ }^{1 *}$, Carmelo Rosales-Guzmán ${ }^{1,2}$, \\ Zhi-Yuan Zhou ${ }^{1,3}$, Dong-Sheng Ding ${ }^{1,3}$, Wei Gao ${ }^{1}$ and Bao-Sen Shi ${ }^{1,3 *}$ \\ ${ }^{1}$ Wang Da-Heng Collaborative Innovation Center, Heilongjiang Provincial Key Laboratory of Quantum Control, Harbin \\ University of Science and Technology, Harbin, China, ${ }^{2}$ Centro de Investigaciones en Óptica, A.C., Colonia Lomas del \\ Campestre, León, Mexico, ${ }^{3}$ Chinese Academy of Sciences (CAS) Key Laboratory of Quantum Information, University of \\ Science and Technology of China, Hefei, China
}

Engineering vector spatial modes of photons is an important approach for manipulating high-dimension photonic states in various quantum optical experiments. In this work, we demonstrate the generation of heralded single photons with well-defined vector spatial modes by using a self-stable polarizing interferometer comprising a spatial light modulator. Specifically, it is shown that, by carefully tailoring and compensating the spatial and temporal amplitudes of manipulated photons, one can exactly convert ultrafast single photons into desired spin-orbit states with an extremely high purity. This compact and robust device provides a versatile way for not only the generation, but also the manipulation and characterization of arbitrary photonic spin-orbit states.

Keywords: vector spatial modes, photonic spin-orbit coupling, single photons, structured light, SLM

\section{INTRODUCTION}

Vectorially structured photons with well-defined vector spatial modes are paraxial light fields whose polarization and transverse modes are non-separable with each other, also known as spinorbit coupled (SOC) states [1-4]. Exploiting photonic SOC states can benefit many quantum technologies based on the photonic platforms, such as high-dimension quantum information [59]. To explore this area, one requires on-demand control of both spin and orbital degrees of freedom (DoFs) of photons namely, having an ability of polarization-dependent spatial mode modulation. The most convenient way for this task is the use of optical geometric phase elements, such as q-plates $[10,11]$. To date, however, all these elements can only provide a phase-only modulation lacking the ability to modulate the spatial complex amplitude of light fields. Therefore, these devices can only work to generate approximate cylindrical vector (CV) modes [12, 13], characterized by a series of propagation-variant radial rings, whose spatial parts are more precisely described as radial-mode degenerated orbital angular momentum (OAM) modes [14]. Noteworthy, to simultaneously shape and control the phase and amplitude of vectorially structured photons, the only feasible way at present is to use spatial light modulators (SLM) combined with various polarizing interferometer schemes.

For example, Christian et al. [15] proposed a self-stable interferometer scheme, based on the combination of a Wollaston prism and an SLM, capable to generate arbitrary vector spatial modes using a complex amplitude modulation implemented on the SLM. Yet, more than $75 \%$ of the photons will be lost due to a double passed beam splitter, and moreover, the calcite made Wollaston prism reduced the beam quality. Recently, Liu et al. [16] reported that using two polarizing Sagnac loops 
as tunable beam displacers and a phase-only SLM realized an arbitrary vector mode generation with a $47 \%$ conversion efficiency. This setup, however, is not compact enough as a SOC module for quantum optical experiments, and they did not demonstrate the generation of propagation invariant modes via this device. More recently, we proposed a compact Digital Micromirror Device (DMD) based setup for a dynamical generation of arbitrary vector modes with a $30 \mathrm{kHz}$ frame rate [17]. Given that DMDs can only realize a binary holographic grating, the photon utilization of this device is too low for quantum experiments.

In this work, we report a compact and robust device for the generation, manipulation, and characterization of vectorially structured photons. To demonstrate its performance, an ultrafast single-photon signal with the $\mathrm{TEM}_{00}$ mode was efficiently converted into a series of vector spatial modes with a high accuracy. Particularly, we show that the purity of prepared SOC states can get extremely close to the theoretical predictions, after compensating the errors in the spatial and temporal amplitudes (compared with target states) of converted photons.

\section{MATERIALS AND METHODS}

Vector spatial modes are theoretical eigen-solutions of the vector paraxial wave equation that corresponds to the propagation of invariant beams with a spatially variant polarization. These vector solutions can also be expressed as a non-separable superposition of orthogonal scalar spatial modes $\psi_{ \pm}$and associated polarizations $\hat{e}_{ \pm}$, which, for instance, in cylindrical coordinates are given by $E(r, \varphi, z)=\alpha \psi_{+}(r, \varphi, z) \hat{e}_{+}+$ $\beta \psi_{-}(r, \varphi, z) \hat{e}_{-}$, where $\alpha$ and $\beta$ are complex probability amplitudes. Importantly, it is possible to represent any spatial mode as a superposition of the OAM carrying modes, e.g., Laguerre-Gauss (LG) modes, that is, vector spatial modes can also be generally regarded as photonic SOC states, and thus, can be simply denoted using the Dirac notation as $\alpha\left|\psi_{+}, \hat{e}_{+}\right\rangle+$ $\beta\left|\psi_{-}, \hat{e}_{-}\right\rangle$. To experimentally generate and control this photonic state, any apparatus must be capable to simultaneously tailor the spatial complex amplitudes of the two spin-dependent spatial modes.

Figure 1 shows a schematic representation of our proposed device, which is a compact self-locking Mach-Zehnder interferometer. The key components comprise of a pair of polarizing beam displacing (PBD) prisms made by fused silica. Compared to a calcite beam displacer (as the one used in [15]), the PBD features a higher transmission, low beam distortion, and the option for a flexible separation-distance. By contrast, the length of a calcite beam displacer with a $5 \mathrm{~mm}$ separation distance will exceed $5 \mathrm{~cm}$, leading to a serious beam distortion and high cost. To experimentally demonstrate the feasibility of our device for tailoring ultrafast single photons, we used a $1.5 \mathrm{~mm}$ type-II PPKTP crystal pumped by a SHG of $795 \mathrm{~nm}$ laser (Toptica TA Pro) to generate the heralded single-photon signal. The photon source has a $5 \mathrm{MHz}$ brightness (pairs/second)

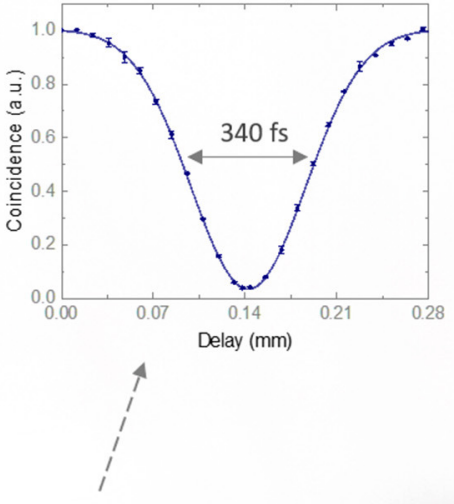

Heralded single photons

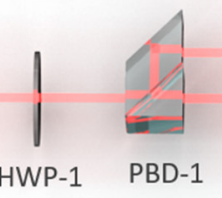

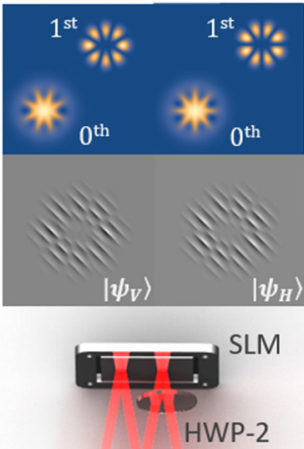

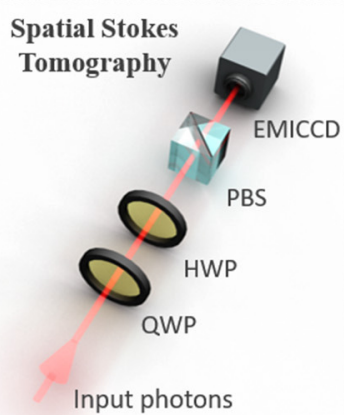

HWP-2

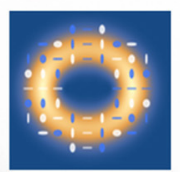

SOC photons

$\mathrm{M}$

M

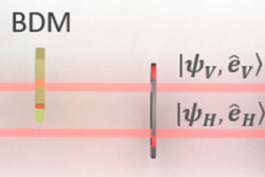

HWP-3
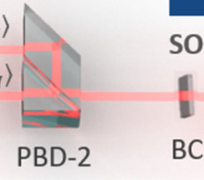

BC

FIGURE 1 | Schematic representation of the experimental setup, where the key components include the polarizing beam displacing prism (PBD), a spatial light modulator (SLM), half-wave plates (HWP), a beam displacement module (BDM), mirrors (M), and a birefringence crystal (BC). The upper-left inset shows the HOM dip of two-photon pairs and the upper-right one shows the setup for the spatial Stokes tomography. 
with a heralding ratio of $90 \%$ (detector excluded). The measured Hong-Ou-Mandel (HOM) dip of the photon pair is shown in the upper-left inset in Figure 1, where a 340 fs FWHM indicates the coherent time of photons is $\sim 240 \mathrm{fs}$. To generate a desired SOC state $\alpha\left|\psi_{H}, \hat{e}_{H}\right\rangle+\beta\left|\psi_{V}, \hat{e}_{V}\right\rangle$, the single-photon signal, which enters from the left side of the setup, was converted into a polarization-path non-separable state, i.e., $\alpha\left|\hat{e}_{H}, 01\right\rangle+\beta\left|\hat{e}_{V}, 10\right\rangle$, by a half-wave plate (HWP-1) and a PBD-1. After that, each single photon was simultaneously sent to two different sections, independently controlled and of a high reflectance (99\%) SLM (Holoeye PLUTO-2-080). In addition, the probability amplitude of photons with $\mathrm{V}$-polarization, i.e., $\beta$, twice passed through the HWP-2 fixed at $45^{\circ}$. This is required because SLMs can only modulate $\mathrm{H}$-polarized light. Hence, by independently tailoring the spatial mode within two polarization subspaces of the photons, they further converted the photons into a three DoFs non-separable state, i.e., $\alpha\left|\psi_{H}, \hat{e}_{H}, 01\right\rangle+\beta\left|\psi_{V}, \hat{e}_{V}, 10\right\rangle$. Finally, HWP-3 and PBD-2 recombined the single photons traveling along the two paths [18], in this way, generating the desired SOC state. Additionally, a single-photon sensitive camera (EMICCD, PI-MAX4) combined with polarizers were used to perform the spatial Stokes tomography on the generated modes [19], as shown in the upper-right inset of Figure 1.

\section{RESULTS}

The purity of the generated SOC states lies with the consistency of the complex amplitude, in both spatial and temporal DoFs, between the prepared and the desired state. Experimentally, realization of this consistency requires high accuracies in orthogonal polarization control, spatial modes generation, and the installation and adjustment of the interferometer. For the polarization control, the fused silica PBD can provide a high enough extinction ratio $(>1,000: 1)$. Therefore, the other two factors are crucial for the generation of the pure states.

The principle of complex amplitude modulation via a phaseonly SLM consists on designing a special phase hologram with blazed grating whose spatial phase-delay and grating depth (efficiency) control the wavefront and the intensity of diffracted light, respectively. The corresponding desired spatial mode is generated along the 1st order diffraction [20]. Note that the input photons are not an ideal plane wave, usually a Gauss mode. Therefore, to generate a well-defined spatial mode, we need to make a correction in the hologram according to the intensity profile of input photons. Figure 2A shows the observed beam profile of input photons before (original intensity, OI) and after (filtered intensity, FI) using the Fourier noise reduction, as well as the case of a maximum photon utilization for generating the $L G_{01}$ mode (target intensity, TI). For comparison, Figure 2B shows the holograms before and after the correction and associated diffraction results. We see that, by using the corrected hologram, an exact $L G_{01}$ mode appears in the 1st order diffraction. Besides, Figure $2 \mathrm{C}$ provides a generation efficiency for various modes, where $L G$ and $I G$ denote Laguerre- and Ince-Gauss modes [2124], respectively. We see that, due to the high reflectance of the used SLM, the measured 1st order diffraction efficiencies for
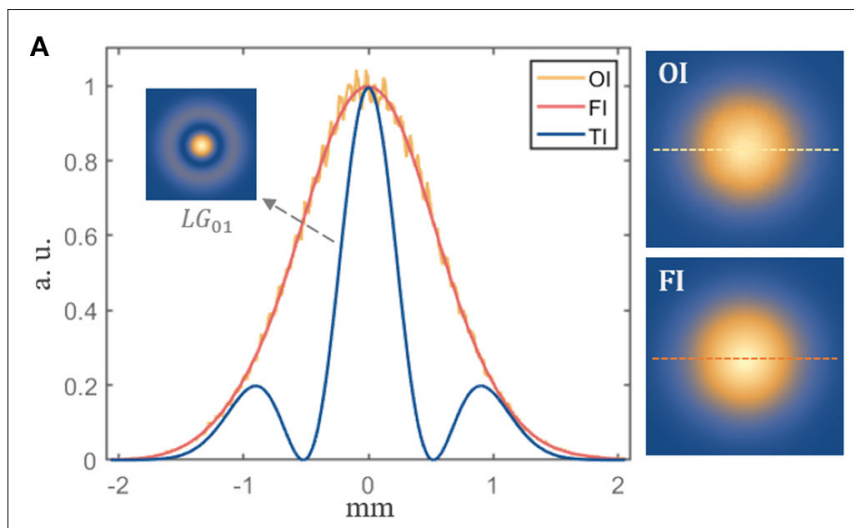

B
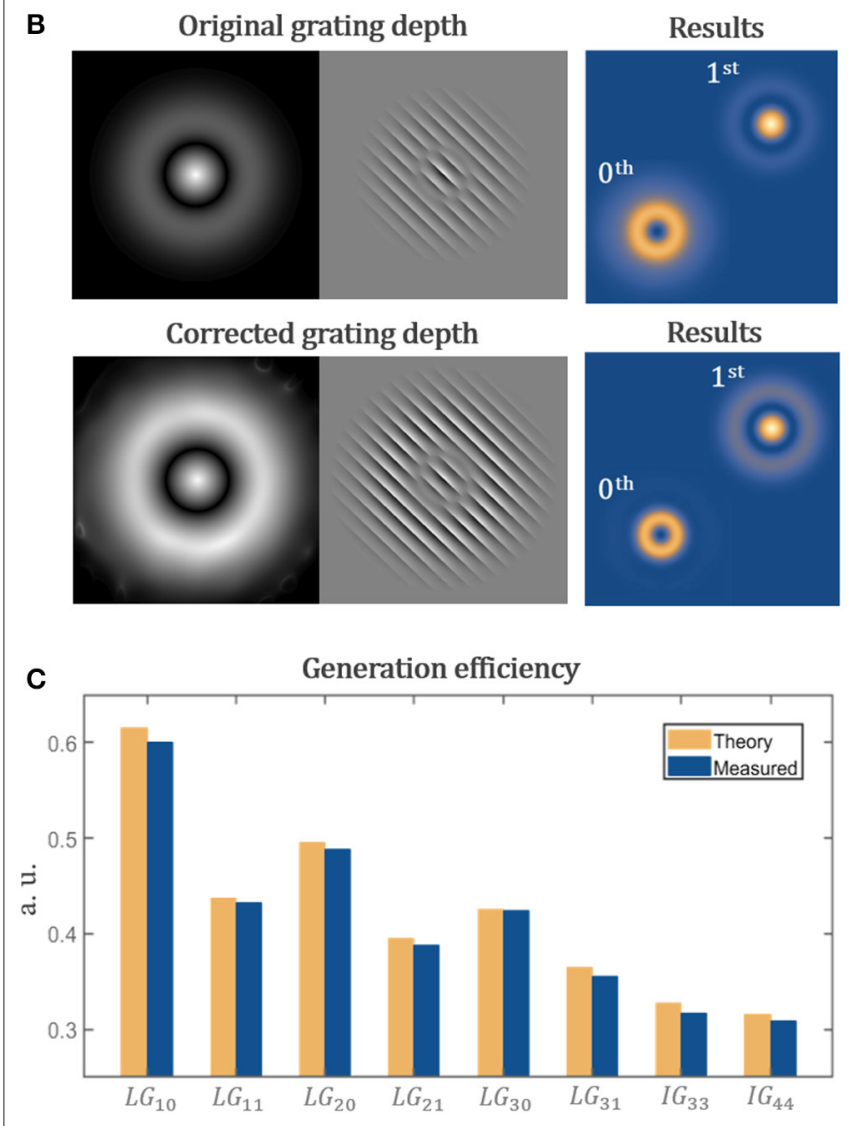

FIGURE 2 | Results of spatial mode generation, (A) characterization of illuminating photons; (B) correction of the hologram for spatial mode generation; (C) generation efficiencies for different spatial modes about the 1st order diffraction.

all cases approach the theoretical limitation. For more details about the generation efficiency of structured Gauss modes, see Rosales-Guzmán and Forbes [20] and Wu et al. [25].

In the interferometer adjustment, it is important to note that there are errors in the separation distance and parallelism of the PBD. Namely, using the prim pairs cannot directly construct a perfect beam separation and combination loop, leading to a dislocation between the optical axis of $\psi_{ \pm}$. To compensate this 


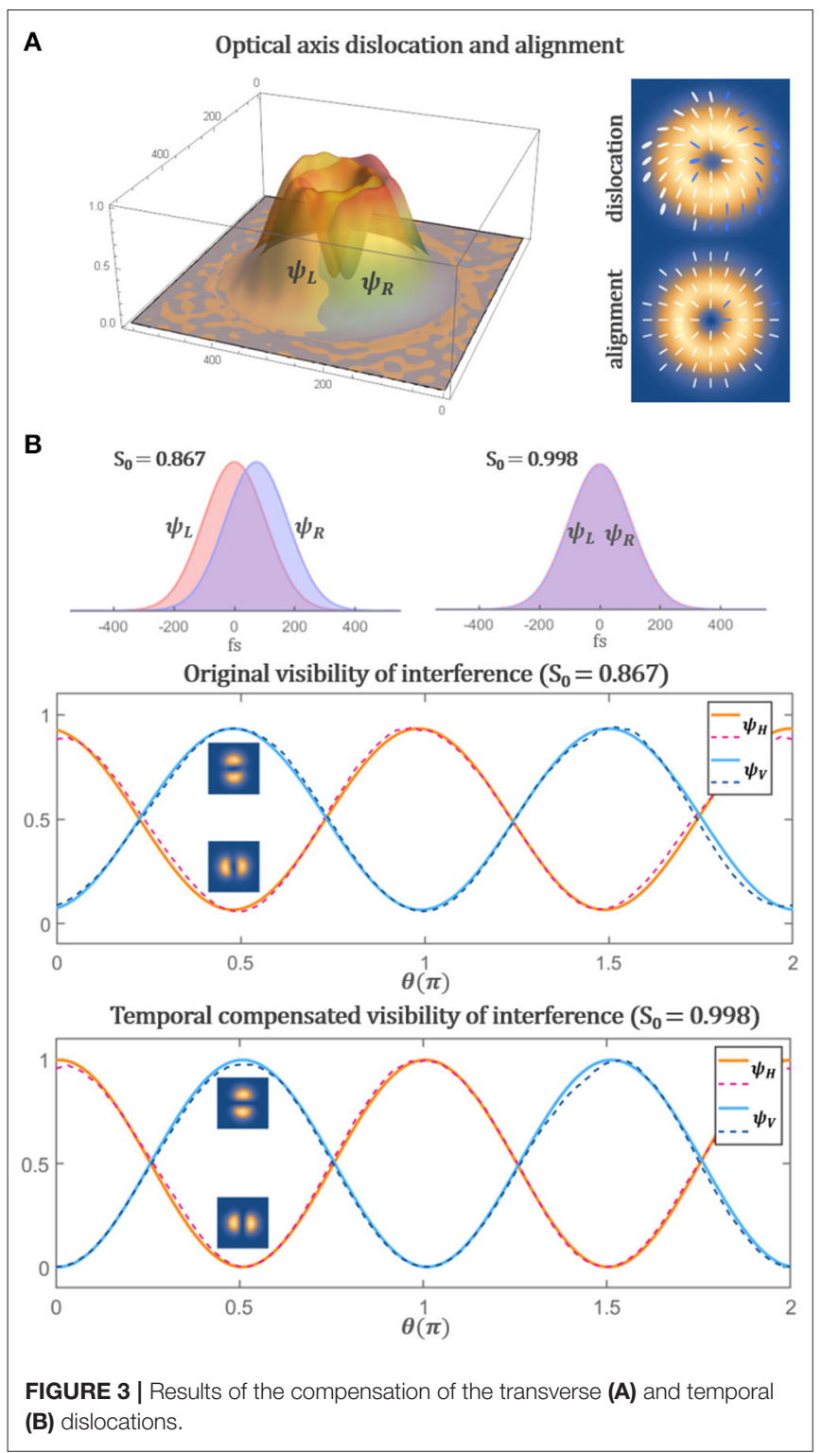

dislocation, two techniques were adopted: (i) a $2 \mathrm{~mm}$ tweaker plate was used as a beam displacement module (BDM) to compensate with the error of PBD in the separation distance; and (ii) fine control the grating period of the arm inserted $\mathrm{BDM}$ so that the error of $\mathrm{PBD}$ in parallelism can be wellcompensated. For comparison, Figure $3 \mathbf{A}$ shows the observed radial polarization mode, i.e., $\sqrt{1 / 2}\left(\left|\psi_{L}, \hat{e}_{L}\right\rangle+\left|\psi_{R}, \hat{e}_{R}\right\rangle\right)$, before and after the compensation of spatial amplitude.

In addition, because the coherent length of input photons is only about $72 \mu \mathrm{m}$, the temporal dislocation between $\psi_{ \pm}$ accumulated in the interferometer should be considered and compensated. The optical path difference (OPD) between the two arms induced by transmission components is small ( $\mu \mathrm{m}$ level), because the thickness of HWP-2 (double passed) and BDM are 1 and $2 \mathrm{~mm}$, respectively. The OPD between two arms mainly originated from the extraordinary reflection angle induced by the
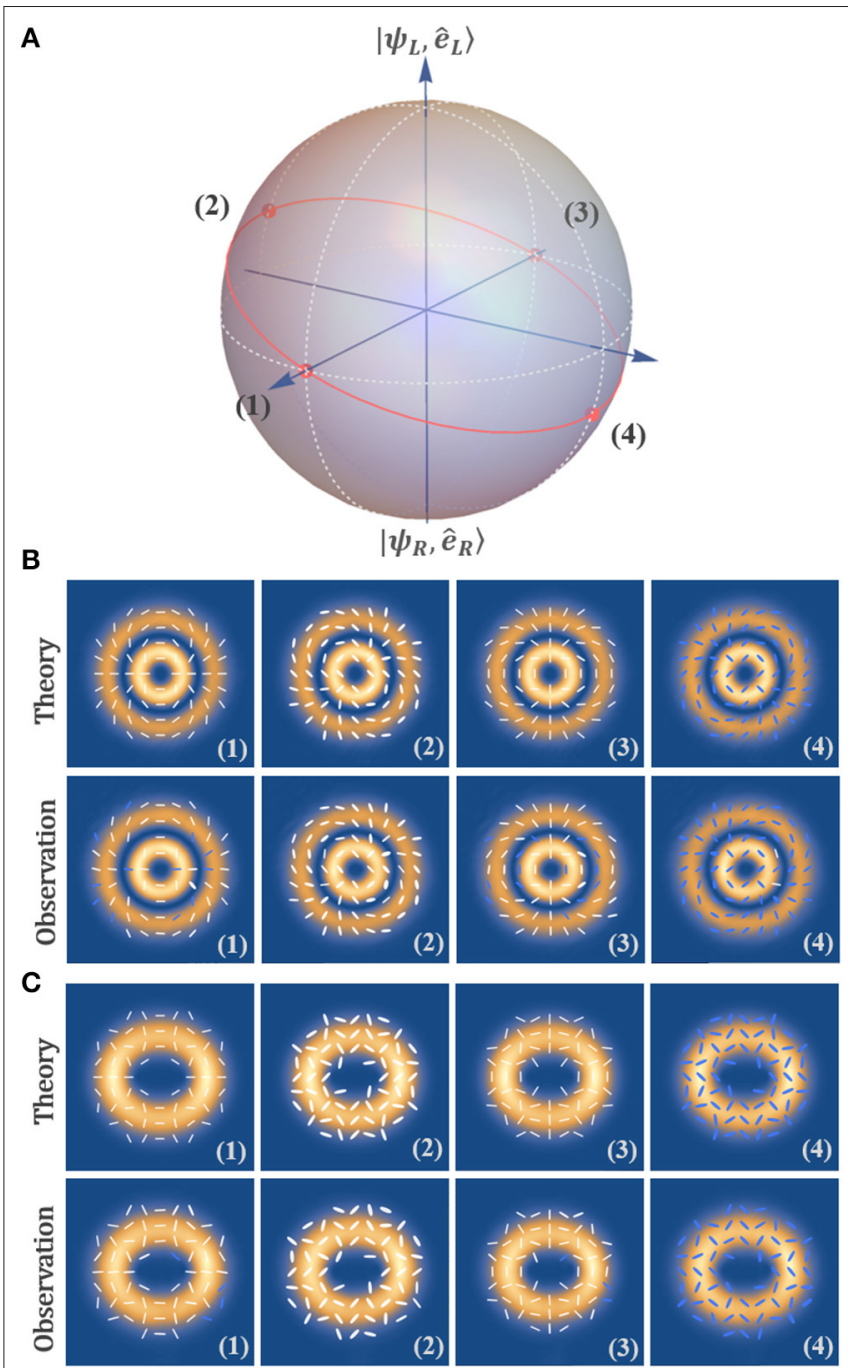

FIGURE 4 | Comparison of the generated and desired SOC states on high-order Poincaré sphere (A) and associated vector profiles $(\mathbf{B}, \mathbf{C})$, where the vector profiles were obtained by the spatial Stokes tomography with a 10 s on-chip accumulation and Fourier noise reduction.

blazed grating. In the experiment, the grating period is $200 \mu \mathrm{m}$, the incident angle is within $5^{\circ}$, and the beam distance is $5 \mathrm{~mm}$. By calculation, we have known that the OPD induced by the blazed grating was $\sim 20 \mu \mathrm{m}$. This temporal dislocation can be easily compensated by passing a birefringence crystal, such as a $0.2-0.3 \mathrm{~mm}$ BBO or KTP crystal.

In order to accurately know the OPD, we measured the state purity of the generated modes in temporal DoF. This purity can be determined via measuring the azimuthal interference visibility of the transverse pattern of a prepared radial-polarization $\mathrm{CV}$ mode in $\hat{e}_{H^{-}}$and $\hat{e}_{V}$-post-selection, i.e., $\psi_{H / V}(\theta)=$ $\sqrt{1 / 2}\left(\left|L G_{+10}\right\rangle \pm e^{i \theta}\left|L G_{-10}\right\rangle\right)$, as shown in Figure 3B. Note that, one can directly obtain the visibility by calculating the average Stokes parameter $\mathrm{S}_{0}$ over the transverse plane, i.e., $\bar{S}_{0}(x, y)$, where $\bar{S}_{0}(x, y)=0$ and 1 correspond to a mixed and pure state, 
respectively, and a detailed theory about this is to be published in recent. We thus have known that the OPD is about $32 \mu \mathrm{m}$ which was obtainable by measuring a 0.867 visibility. Then, we inserted a $300 \mu \mathrm{m} \mathrm{BBO}$ crystal at the output port to compensate with this temporal dislocation. We see that, after the temporal dislocation compensation, a near perfect pure state with an extremely high 0.998 visibility was achieved.

After finishing adjustment of the interferometer, we chose two groups of vectorially structured photons, i.e., four red points on the high-order Poincaré sphere for each group shown in Figure 4A, generated by the device to show its performance. Specifically, the high-order Poincaré sphere in the first group is spanned by $\left|L G_{+21}, \hat{e}_{L}\right\rangle$ and $\left|L G_{-21}, \hat{e}_{L}\right\rangle$, while in the other one, $\psi_{L / R}=\sqrt{1 / 2}\left(\left|I G_{44}^{0}\right\rangle \pm i\left|I G_{44}^{e}\right\rangle\right)$ with an ellipticity of $\varepsilon=1$. We see that the vector profiles of the prepared SOC states, shown in the second row of Figures $4 B, C$, excellently coincide with the theoretical desired states. Particularly, the modes in the second group are the vector Ince-Gauss modes that were proposed in our recent work [24]. These modes are propagation invariant and thus can be regarded as eigen solutions of the vector paraxial wave equation in the corresponding elliptical cylindrical coordinates. Besides, the vector profiles were obtained by spatial Stokes tomography with a $10 \mathrm{~s}$ on-chip accumulation, namely, the reconstruction of each file requires 40 s to acquire $\psi_{L}, \psi_{R}, \psi_{H}$, and $\psi_{D}$, respectively. See Zhou et al. [26] for more details about the imaging. Even so, all the profiles still coincide well with the theory. This indicates that the relative phase of the two paths (i.e., the intramodal phase of vector modes) was highly stable during the date acquisition, which is crucial for quantum experiments.

\section{DISCUSSION AND CONCLUSION}

We have demonstrated the superiority of our device for the generation of arbitrary high-purity SOC states of single photons. It is noteworthy that this device can also be used for shaping entangled photons., for example, converting a polarization entanglement into a hyperentanglement and vice versa. Besides,

\section{REFERENCES}

1. Chen J, Wan C-H, Zhan Q-W. Vectorial optical fields: recent advances and future prospects. Sci Bull. (2018) 63:54. doi: 10.1016/j.scib.2017.12.014

2. Rosales-Guzmán C, Ndagano B, Forbes A. A review of complex vector light fields and their applications. J Opt. (2018) 20:123001. doi: 10.1088/2040-8986/aaeb7d

3. Cardano F, Marrucci L. Spin-orbit photonics. Nat Photon. (2015) 9:776. doi: 10.1038/nphoton.2015.232

4. Borges CVS, Hor-Meyll M, Huguenin JAO, Khoury AZ. Belllike inequality for the spin-orbit separability of a laser beam. Phys Rev A. (2010) 82:033833. doi: 10.1103/PhysRevA.82. 033833

5. Norrman A, Friberg T, Leuchs G. Vector-light quantum complementarity and the degree of polarization. Optica. (2020) 7:93-7. doi: 10.1364/OPTICA.7.000093

6. Souza ER, Borges CVS, Khoury AZ, Huguenin JAO, Aolita L, Walborn SP. Quantum key distribution without a shared reference frame. Phys Rev A. (2008) 77:032345. doi: 10.1103/PhysRevA.77.032345 in the classical domain, this device has also an advantage in the efficiency and accuracy for shaping vector beam or pulses compared to previous works $[15,16]$. In summary, we proposed a SLM-based self-locking polarization interferometer for shaping vectorially structured photons. By theoretical analysis and experimental observation, we show that this compact and robust device can efficiently convert ultrafast photons into the desired SOC states with an extremely high purity. This programmable interferometer can also work as a versatile photonic SOC modulator for the manipulation and characterization of photonic SOC states. During the preparation of this paper, we found a pre-print paper reporting the similar topic, where a more compact prism-based interferometer was adopted [27]. However, due to the absence of SLM, this prism interferometer can only work for controlling the CV modes. Namely, this prism-based interferometer can be regarded as an integrated version of the Sagnac loop used in our recent experiments [28-30].

\section{DATA AVAILABILITY STATEMENT}

The original contributions presented in the study are included in the article/supplementary material, further inquiries can be directed to the corresponding authors.

\section{AUTHOR CONTRIBUTIONS}

Z-HZ conceived the project. H-JW and B-SY conducted the experiment. Z-HZ wrote the manuscript. Z-HZ and B-SS supervised the project. All authors contributed to the discussions during its preparation.

\section{FUNDING}

This work was funded by the National Natural Science Foundation of China (Grant Nos. 11934013, 62075050, and 61975047).
7. D’Ambrosio V, Nagali E, Walborn SP, Aolita L, Slussarenko S, Marrucci L, et al. Complete experimental toolbox for alignment-free quantum communication. Nat Commun. (2012) 3:961. doi: 10.1038/ncomms1951

8. Zhang W, Ding DS, Dong MX, Shi S, Wang K, Liu S-L, et al. Experimental realization of entanglement in multiple degrees of freedom between two quantum memories. Nat Commun. (2016) 7:13514. doi: $10.1038 /$ ncomms 13514

9. Sit F, Bouchard R, Fickler J, Bischoff G, Larocque H, Heshami K, et al. Highdimensional intracity quantum cryptography with structured photons. Optica. (2017) 4:1006-10. doi: 10.1364/OPTICA.4.001006

10. Marrucci L, Karimi E, Slussarenko S, Piccirillo B, Santamato E, Nagali E, et al. Spin-to-orbital conversion of the angular momentum of light and its classical and quantum applications. J Opt. (2011) 13:064001. doi: 10.1088/2040-8978/13/6/064001

11. Chen P, Wei B-Y, Hu W, Lu Y-Q. Liquid-crystal-mediated geometric phase: from transmissive to broadband reflective planar optics. Adv Mater. (2019) 32:1903665 doi: 10.1002/adma.201903665

12. Zhan Q. Cylindrical vector beams: from mathematical concepts to applications. Adv Opt Photon. (2009) 1:1. doi: 10.1364/AOP.1.000001 
13. Milione G, Sztul HI, Nolan DA, Alfano RR. Higher-order poincaré sphere, stokes parameters, and the angular momentum of light. Phys Rev Lett. (2011) 107:053601. doi: 10.1103/PhysRevLett.107.053601

14. Karimi E, Zito G, Piccirillo B, Marrucci L, Santamato E, HypergeometricGaussian modes. Opt Lett. (2007) 32:3053. doi: 10.1364/OL.32.003053

15. Christian M, Alexander J, Severin F, Stefan B, Monika R-M, Tailoring of arbitrary optical vector beams. New J Phys. (2007) 9:78. doi: 10.1088/1367-2630/9/3/078

16. Liu S, Qi S, Zhang Y, Li P, Wu D, Han L, et al. Highly efficient generation of arbitrary vector beams with tunable polarization, phase, and amplitude. Photon Res. (2018) 6:228-33. doi: 10.1364/PRJ.6.000228

17. Rosales-Guzmán C, Hu X, Selyem A, Moreno-Acosta P, franke-Arnold S, Ramos-Garcia R, et al. Polarisation-insensitive generation of complex vector modes from a digital micromirror device. Sci Rep. (2020) 10:10434. doi: 10.1038/s41598-020-66799-9

18. Zhou Z-Y, Zhu Z-H, Liu S-L, Li Y-H, Shi S, Ding D-S, et al. Quantum twisted double-slits experiments: confirming wavefunctions' physical reality. Sci Bull. (2017) 62:1185. doi: 10.1016/j.scib.2017.08.024

19. Yu BS, Wu H-J, Yang H-R, Gao W, Rosales-Guzman C, Shi B-S, Zhu ZH. Full characterization of spin-orbit coupled photons via spatial-Stokes measurement. arXiv:1907.04035.

20. Rosales-Guzmán C, Forbes A. How to Shape Light with Spatial Light Modulators. SPIE Press Book (2017). doi: 10.1117/3.2281295

21. Bandres MA, Gutiérrez-Vega JC. Ince-Gaussian beams. Opt Lett. (2004) 29:144-6. doi: 10.1364/OL.29.000144

22. Plick WN, Krenn M, Fickler R, Ramelow S, Zeilinger A. Quantum orbital angular momentum of elliptically symmetric light. Phys Rev A. (2013) 87:033806. doi: 10.1103/PhysRevA.87.033806

23. Yang H-R, Wu H-J, Gao W, Rosales-Guzmán C, Zhu Z-H. Parametric upconversion of Ince-Gaussian modes. Opt Lett. (2020) 45:3034. doi: 10.1364/OL.393146

24. Li Y, Hu X-B, Perez-Garcia B, Zhao B, Gao W, Zhu Z-H, et al. Classically entangled Ince-Gaussian modes. Appl. Phys Lett. (2020) 116:221105. doi: $10.1063 / 5.0011142$
25. Wu H-J, Mao L-W, Yang Y-J, Rosales-Guzmán C, Gao W, Shi B$\mathrm{S}$, et al. Radial modal transition of Laguerre-Gauss modes in secondharmonic generation: towards the full-field selection rule of spatial modes. Phys Rev A. (2020) 101:063805. doi: 10.1103/PhysRevA.101. 063805

26. Zhou J, Liu S, Qian H, Li Y, Luo H, Wen S, et al. Metasurface enabled quantum edge detection. Sci Adv. (2020) 6:eabc4385. doi: 10.1126/sciadv.abc4385

27. Ren Z-C, Cheng Z-M, Wang X-L, Ding J, Wang H-T. Polarization interferometric prism: a versatile tool for generation of vector fields, measurement of topological charges and implementation of a spin-orbit Controlled-Not gate. arXiv:2012.12584

28. Wu H-J, Yang H-R, Rosales-Guzmán C, Gao W, Shi B-S, Zhu Z-H. Vectorial nonlinear optics: Type-II second-harmonic generation driven by spin-orbit-coupled fields. Phys Rev A. (2019) 100:053840. doi: 10.1103/PhysRevA.100.053840

29. Wu H-J, Zhou Z-Y, Gao W, Shi B-S, Zhu Z-H. Dynamic tomography of the spin-orbit coupling in nonlinear optics. Phys Rev A. (2019) 99:023830. doi: 10.1103/PhysRevA.99.023830

30. Wu H-J, Zhao B, Rosales-Guzmán C, Gao W, Shi B-S, Zhu ZH. Spatial-polarization-independent parametric up-conversion of vectorially structured light. Phys Rev Applied. (2020) 13:064041. doi: 10.1103/PhysRevApplied.13.064041

Conflict of Interest: The authors declare that the research was conducted in the absence of any commercial or financial relationships that could be construed as a potential conflict of interest.

Copyright (C) 2021 Wu, Yu, Zhu, Rosales-Guzmán, Zhou, Ding, Gao and Shi. This is an open-access article distributed under the terms of the Creative Commons Attribution License (CC BY). The use, distribution or reproduction in other forums is permitted, provided the original author(s) and the copyright owner(s) are credited and that the original publication in this journal is cited, in accordance with accepted academic practice. No use, distribution or reproduction is permitted which does not comply with these terms. 